\title{
T-NOVA: Network Functions as-a-Service over Virtualised Infrastructures
}

\author{
George Xilouris ${ }^{1}$, Michail-Alexandros Kourtis ${ }^{1}$, Michael J. McGrath ${ }^{2}$, Vincenzo Riccobene ${ }^{2}$, \\ Guiseppe Petralia ${ }^{2}$, Evangelos Markakis ${ }^{3}$, Evangelos Palis ${ }^{3}$, Alexiou Georgios ${ }^{3}$, Georgios Gardikis ${ }^{4}$, \\ Jordi F. Riera ${ }^{5}$, Aurora Ramos ${ }^{6}$, José Bonnet ${ }^{7}$ \\ ${ }^{1}$ National Centre of Scientific Research "Demokritos", Athens, Greece \\ ${ }^{2}$ Intel Labs Europe, Leixlip, Co. Kildare, Ireland \\ ${ }^{3}$ Technological Educational Institute of Crete, Heraklion, Greece \\ ${ }^{4}$ R\&D Department, Space Hellas S.A. Athens, \\ ${ }^{5}$ Distrib. Applic. \& Networks Area i2CAT Found., Barcelona, Spain \\ ${ }^{6}$ ATOS Spain S.A., Spain \\ ${ }^{7}$ Portugal Telecom Inovaçao e Sistemas, Aveiro, Portugal
}

\begin{abstract}
The EU funded FP7 project T-NOVA, with the specific goal of accelerating the evolution of NFV, proposes an open architecture to provide Virtual Network Functions as a Service (VNFaaS), together with a dynamic, and flexible platform for the management of Network Services (NSs) composed by those Virtual Network Functions (VNFs). The proposed architecture allows operators to deploy distinct virtualized network functions, not only for their internal operational needs, but also to offer them to their customers, as value-added services. Virtual network appliances (e.g. gateways, proxies or even traffic analyzers) can be provided on-demand, eliminating the need to acquire, install, and maintain specialized hardware at customer premises. This demo illustrates work carried out on the deployment of a VNF on a Network Function Virtualization Infrastructure (NFVI) using resource aware scheduling methods to ensure optimal use of resources and performance while we focus also on business-related and customer front-end aspects, i.e. how the stakeholders interact with the services, which T-NOVA identifies as the marketplace layer.
\end{abstract}

Keywords-NFV, VNF, Marketplace, virtualisation, NFaaS, T-NOVA;

\section{EXTENTED ABSTRACT}

The EU funded FP7 T-NOVA project is focused on realizing the concept of Network Functions as a Service (NFaaS) by designing and implementing an integrated management architecture, for the automated provision, management, monitoring and optimization of VNFs over Network/IT infrastructures [1]. TNOVA project implements an open architecture to offer Virtual Network Functions (VNFs) as a service (VNFaaS), enabled via a flexible management core (the T-NOVA Orchestrator) and supported by a multiactor transaction platform (the T-NOVA
Marketplace). The latter facilitates the involvement of diverse actors in the NFV scene and aims in attracting new market entrants. T-NOVA "NFV Marketplace", Network Functions by several developers can be published, brokered and traded [2].

The motivation for the proposed demo is on one hand to illustrate the interactions between the actors at the high layers of T-NOVA architecture i.e Marketplace and Orchestrator and the information that has to be exchanged. On the other hand to demonstrate initial work by the T-NOVA project to address some of the limitations of cloud compute environments with respect to scheduling of virtualized resources to host VNFs in a performance manner. Additionally, Orchestration mechanisms as Service Mapping, Management GUI and Infrastructure Repository and monitoring aspects are also demonstrated.

This demo illustrates how an Network Service (NS) composed by a single VNF (in this case a network traffic classification VNF) can exploit the platform requirements specification contained in an ETSI ISG NFV VNF descriptor (VNFD) accompanying it (extended by T-NOVA), in order to deploy and appropriately configure the virtualized infrastructure resources in a performant manner as proposed by the project. The demonstrated scenario involves T-NOVA SP that will be able to acquire VNFs, interacting with different NF developers through a brokerage/trading procedure not only for its own needs but also to offer composed NSs to its own customers. For this, SLA and billing information between SP and FPs will be managed. SP will define their own SLA commitments based on the SLAs provided by NF providers and billable items will be shared among customers, SP and NF providers. Steps where the VNF and NS descriptors are created is also demonstrated. Upon deployment of the selected NS the Orchestrator parses 
the information included in the VNFD into corresponding metadata, which is then used to dynamically construct an Openstack Heat template. The template is then used in an OpenStack Cloud environment to automatically instantiate the required virtualized resources with specific platforms features that cannot be predictably provisioned using OpenStack's current scheduling mechanism. Finally a side-to-side comparison of the performance of the virtual Traffic Classification VNF's running on 'standard Virtual Machines' (VMs) versus VMs with features to improve packet processing performance is demonstrated using real-time instrumentation.

\section{EQUIPMENT USED FOR DEMONSTRATION}

The demo architecture shown in Figure 1 comprises of an OpenStack Juno based cloud environment. The cloud environment comprises of a controller and two compute nodes, and a traffic generator connected on the same network domain through a 10 Gbps switch. The compute node configuration enables the VMs to use both vNICs connected to Open virtual Switch $(\mathrm{OvS})$ in the form of software-assisted solution and physical NICs with PCIe passthrough/SR-IOV functionality in the form of a hardware-assisted solution.

On the same host as the controller, a simulated Orchestrator is running which receives a deployment request (which includes an ETSI compliant VNFD) and converts it into metadata representing the platform deployment requirements of the VNF, which is used to dynamically generate a Heat template. The template orchestrates the setup SR-IOV ports, deployment of Traffic Classification VNF Components (VNFCs) and configuration of the VNFCs The VNF used in this demo comprises of two VNFCs, namely the DPI engine and the Classification and Forwarding functionality. The VNFCs are implemented and contained in the form of two VMs. For the purpose of the demo, two different versions of the VNFD are utilized: the first version contains no platform specific features allowing the Orchestrator request a 'standard' deployment (based on OvS) of the DPI; the second version contains specific platform features i.e. an SRIOV capable NIC which must be available on the physical sever were the VMs are hosting the vDPI.

Once the two different instances of the Traffic Classification VNF are running, traffic is generated by the packet generator and sent to both the instances. The performance of both deployments is compared using real-time display of parametric data from the VMs captured using embedded instrumentation agents in the VMs to highlight differences in packet processing performance.

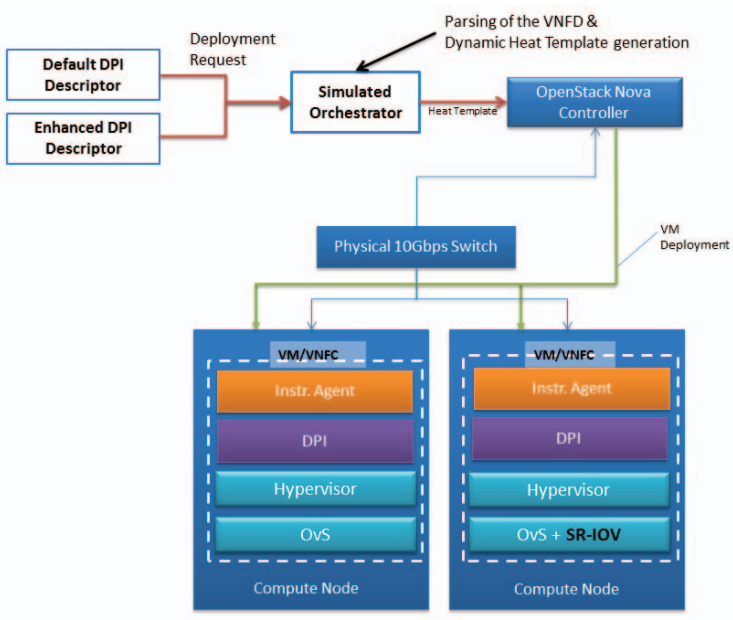

Fig. 1. Architecture of the demo testbed.

The demonstration is based partially on components available by remote access connection to one of the TNOVA Pilot sites and on software components and demonstration software that is installed on laptops and PCs that will be shipped to the venue. In this context the sole requirement is Internet connectivity. However as a contingency plan, parts of the demo will be also possible to be demonstrated in simulated fashion.

\section{SPACE AND SETUP TIME}

The described demo has been presented in various venues in an early version, hence experience on the setup and requirements has been acquired. The anticipated space requirements are no more than space occupied by 3-4 laptops. The required setup time is no more than 30 minutes - 1hour, depending on the setup. [3] references a URL for accessing interactive version of the demo, along with some information.

\section{ACKNOWLEDGMENT}

This work was undertaken under the Information Communication Technologies, EU FP7 T-NOVA project, which is partially funded by the European Commission under the grant 619520.

\section{REFERENCES}

[1] T-NOVA Consortium, Deliverable 2.21- Overall system architecture and Interfaces, June 2014, on-line:http://www.tnova.eu/results

[2] G. Xilouris et. al, T-NOVA: A Marketplace for Virtualized Network Functions, European Conference on Networks and Communications, Bologna, Italy, June 2014.

[3] On-line DEMO web-page, T-NOVA Consortium, http://demo.t-nova.eu 